INPLASY

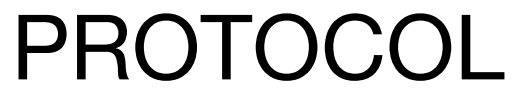

To cite: Huang et al. Risk factors for postoperative intestinal obstruction in colorectal cancer. Inplasy protocol 202140125. doi: 10.37766/inplasy2021.4.0125

Received: 25 April 2021

Published: 25 April 2021

Corresponding author: Huang Yishen

385656332@qq.com

Author Affiliation: Graduate School of Hebei Medical University

Support: No.

Review Stage at time of this submission: Data extraction.

Conflicts of interest:

None declared.

\section{Risk factors for postoperative intestinal obstruction in colorectal}

\section{cancer}

Review question / Objective: P: Participant, Patients with colorectal cancer underwent surgery $E$ : exposure, postoperative ileus of colorectal cancer C: Comparison, no postoperative ileus after colorectal cancer surgery 0 : Outcome, Risk factors for postoperative intestinal obstruction in patients with colorectal cancer.

Condition being studied: Intestinal obstruction in colorectal cancer.

Information sources: China knowledge Network, China Biomedical Database, Wanfang Database, VIP Chinese Medical Journal Database, PubMed, Web of Science, Embase and The Cochrane Library databases.

INPLASY registration number: This protocol was registered with the International Platform of Registered Systematic Review and Meta-Analysis Protocols (INPLASY) on 25 April 2021 and was last updated on 25 April 2021 (registration number INPLASY202140125).

\section{INTRODUCTION}

Review question / Objective: P: Participant, Patients with colorectal cancer underwent surgery $\mathrm{E}$ : exposure, postoperative ileus of colorectal cancer
C: Comparison, no postoperative ileus after colorectal cancer surgery 0 : Outcome, Risk factors for postoperative intestinal obstruction in patients with colorectal cancer. 
Condition being studied: Intestinal obstruction in colorectal cancer.

\section{METHODS}

Participant or population: Patients with colorectal cancer underwent surgery.

Intervention: Postoperative ileus of colorectal cancer.

Comparator: No postoperative ileus after colorectal cancer surgery.

Study designs to be included: case control study.

Eligibility criteria: It is consistent with the diagnosis of postoperative intestinal obstruction. Inclusion criteria (1) the original literature published at home and abroad on the risk factors of postoperative intestinal obstruction in patients with colorectal cancer is complete and provides an advantage over (OR) and $95 \%$ confidence interval $(95 \% \mathrm{Cl})$; (2) NewcastleOttawa scale (the NewcastleOttawaScale,NOS) score $\geq 5$ stars; (3) consistent with the diagnosis of postoperative intestinal obstruction. . 2 exclusion criteria: (1) study cases of intestinal obstruction 30 days after resection of colorectal cancer; (2) review, systematic review, basic research or conference papers; (3) statistical errors in repeatedly published literature, similar literature or literature; (4) the study did not involve risk factors or did not provide relevant data; (5) animal experiments.

Information sources: China knowledge Network, China Biomedical Database, Wanfang Database, VIP Chinese Medical Journal Database, PubMed, Web of Science, Embase and The Cochrane Library databases.

Main outcome(s): Risk factors for postoperative intestinal obstruction in patients with colorectal cancer.

Quality assessment / Risk of bias analysis: the Newcastle-Ottawa Scale.
Strategy of data synthesis: Meta analysis was performed by ReviewManager5.3 and STATA 16.0 , and $O R$ and $95 \% \mathrm{Cl}$ were combined to evaluate the relationship between risk factors and postoperative intestinal obstruction of colorectal cancer. If the heterogeneity test is $P \geq 0.1$ and $12 \leq$ $50 \%$, it is suggested that the studies are homogeneous and the fixed effect model (Fixed-effect model, FEM) is used to analyze. If $P<0.1$ and $I 2>50 \%$, it indicates that there is heterogeneity. After excluding the studies with obvious heterogeneity, if there is still more than moderate heterogeneity, random effect model (Random-effects mode, REM) analysis should be used, and subgroup analysis should be used to explore the sources of heterogeneity; otherwise, FEM should be used for analysis.

Subgroup analysis: In the study of obvious heterogeneity, subgroup analysis and other methods were used to explore the source of heterogeneity.

Sensitivity analysis: The ReviewManager5.3 software is used to analyze the sensitive situation of the article by deleting the change of the amount of effect after one of the articles is deleted.

Country(ies) involved: China.

Keywords: colorectal cancer; postoperative ileus; risk factors; Meta-analysis; Logistic model.

Contributions of each author:

Author 1 - Huang Yishen.

Author 2 - Li Zhongxin.

Author 3 - Jia Yitao. 